Pacific Journal of Mathematic 


\section{A FAMILY OF COUNTABLE HOMOGENEOUS GRAPHS}

\section{WARD HENSON}

Let $\mathscr{C}$ be the class of all countable graphs and let $\mathscr{K}_{p}$ be the class of all members of $\mathscr{K}^{C}$ which have no complete subgraphs of cardinality $p$. R. Rado has constructed a graph $U$ which is universal for $\mathscr{K}$. In this paper $U$ is shown to be homogeneous, in the sense of Fraissé. Also a simple construction is given of a graph $G_{p}$ which is homogeneous and universal for $\mathscr{K}_{p}$ (for each $p \geqq 3$ ) and the structure of these graphs is investigated.

It is shown that if $H$ is an infinite member of $\mathscr{K}_{p}$ then $H$ can be embedded in $G_{p}$ in such a way that every automorphism of $H$ extends uniquely to an automorphism of $G_{p}$. A similar result holds for $U$. Also, $U$ and $G_{3}$ have single-orbit automorphisms, while if $p>3$, then $G_{p}$ has no such automorphism. Finally, a result concerning vertex colorings of the graphs $G_{p}$ is proved and used to give a new proof of a Theorem of Folkman on vertex colorings of finite graphs.

1. A graph $G$ is a relational structure which consists of a nonempty set $|G|$ of vertices and an irreflexive, symmetric binary relation $R(G)$ on $|G|$. If $A \subset|G|$ is nonempty, let $G \mid A$ denote the induced subgraph of $G$ which has vertex set $A$. Write $H \subset G$ to mean that $H$ equals $G \mid A$ for some $A \subset|G|$. An embedding of $H$ into $G$ is an isomorphism of $H$ onto an induced subgraph of $G$. If such an embedding exists we say that $G$ admits $H$. If $G$ and $H$ are isomorphic we write $G \cong H$.

The complement graph of $G$ is denoted by $\bar{G}$. $K_{p}$ denotes a complete graph with $p$ vertices ( $p$ an integer $\geqq 1$.) For each $v \in|G|, G^{v}$ denotes the induced subgraph of $G$ which has vertex set

$$
\{w \mid(w, v) \in R(G)\} \text {. }
$$

(The valence subgraph determined by $v$.) The induced subgraph of $G$ obtained by removing a vertex $v$ will be designated by $G-v$. The cardinality of the vertex set $|G|$ will be denoted by $c(G) . \quad Z$ denotes the set of all the integers and $N$ the set of nonnegative integers.

The study of homogeneous relational structures was begun by Fraissé [4] as an attempt to generalize certain familiar properties of the ordering of the rational numbers. This study was continued in a very general setting by Jónsson [6 and 7] and by Morley and 
Vaught [8]. The basic properties of homogeneous graphs needed in this paper may be summarized as follows.

Definition 1.1. A graph $G$ is homogeneous if whenever $H \subset G$ and $c(H)<c(G)$, every embedding of $H$ into $G$ can be extended to an automorphism of $G$.

THEOREM 1.2. An infinite graph $G$ is homogeneous $\longleftrightarrow$ whenever $H \subset G, c(H)<c(G)$ and $v \in|H|$, every embedding of $H-v$ into $G$ can be extended to an embedding of $H$ into $G$.

Theorem 1.3. Let $G$ be an infinite homogeneous graph.

(a) Suppose $c(H)=c(G)$ and $G$ admits every graph $K \subset H$ for which $c(K)<c(H)$. Then $G$ admits $H$.

(b) If $H$ is homogeneous, $c(H)=c(G)$ and $G$ and $H$ admit exactly the same graphs of cardinality $<c(G)$, then $H \cong G$.

In case $G$ is a countably infinite graph, as will be true in this paper, Definition 1.1 comes from [4]; in that case, Theorem 1.2 is [4, Theorem 5.5] and Theorem 1.3 is [4, Theorems $V$ and 5.4]. In general, $G$ is homogeneous in the sense of Definition 1.1 if and only if $G$ is $\mathscr{\mathscr { L }}$-homogeneous in the sense of [7] and [8], where $\mathscr{K}$ is the class of all graphs; here Theorems 1.2 and 1.3 are included in [8, Theorems 2.3 and 2.5]. (It should be noted that in [8], and in model theory generally, "homogeneous" is used in a different, weaker sense. This should cause no confusion here, since only the meaning which agrees with [4] will be used.)

Rado's graph $[9,10]$ is universal among countable graphs by virtue of satisfying the condition

(A) if $F_{1}, F_{2}$ are disjoint, finite sets of vertices of $G$, then there is another vertex which is connected in $G$ to every member of $F_{1}$ and to no member of $F_{2}$.

THEOREM 1.4. Any graph $G$ (with $c(G)=\boldsymbol{\aleph}_{0}$ ) which satisfies condition (A) is homogeneous. Moreover, any two such graphs are isomorphic.

Proof. Rado [10] showed that any graph which satisfies (A) must admit every finite graph. Thus the second statement follows from the first by Theorem 1.3.b.

Let $G$ be a graph which satisfies (A) and $c(G)=\boldsymbol{\aleph}_{0}$. We prove that $G$ is homogeneous by showing that it satisfies the condition in Theorem 1.2. Suppose $H \subset G$ and $c(H)<c(G)$, so that $H$ is finite. 
Let $v \in|H|$ and assume $f$ is an embedding of $H-v$ into $G$. Let $F_{1}=f\left(\left|H^{v}\right|\right)$ and $F_{2}=\operatorname{Range}(f)-F_{1}$. There is a vertex $w$ in $|G|$ which is connected to every member of $F_{1}$ and to no member of $F_{2}$. It follows that letting $f(v)=w$ extends $f$ to an embedding of $H$ into $G$, completing the proof.

We will designate by $U$ a graph (isomorphic to Rado's graph) which is constructed as follows. Let $\left\{P_{n} \mid n \in N\right\}$ be an enumeration of the finite subsets of $N$, each one occurring infinitely often. Choose a sequence $v_{0}<v_{1}<\cdots$ in $N$ which satisfies $v_{n}>\max \left(P_{n}\right)$ for all $n \in N$. To define $U$ let $|U|=N$ and let $R(U)$ consist of all pairs of vertices of the form $\left(w, v_{n}\right)$ or $\left(v_{n}, w\right)$ where $w \in P_{n}$ and $n \in N$. Then $U$ satisfies the following strong form of $(A)$.

$\left(\mathrm{A}^{\prime}\right)$ if $F \subset|U|$ is finite, then there exist arbitrarily large $v$ in $|U|$ which satisfy

$$
F=\{w \mid w<v \quad \text { and } \quad(w, v) \in R(U)\} .
$$

In particular, $U$ satisfies (A) and is thus isomorphic to Rado's graph, by Theorem 1.4. (Note that Rado's graph itself does not satisfy $\left(\mathrm{A}^{\prime}\right)$.)

REMARK. In [2] Erdös and Renyi put a natural probability measure on the set of all graphs with vertex set $N$, and show that the measure of the set of such graphs which satisfy condition (A) is 1. They conclude from this that almost all graphs with vertex set $N$ have a nontrivial automorphism. In fact the stronger result, that almost all such graphs are isomorphic to $U$, follows from Theorem 1.4 .

CoROLlary 1.5. (a) $\bar{U} \cong U$

(b) if $|U|=A_{1} \cup \cdots \cup A_{n}$ and $A_{1}, \cdots, A_{n}$ are pairwise disjoint, then $U \mid A \cong U$ for some $j=1, \cdots, n$.

Proof. (a) $\bar{U}$ obviously satisfies condition (A).

(b) It suffices to consider the case $n=2$.

Suppose $|U|=A \cup A^{\prime}$ and $A \cap A^{\prime}=\varnothing$, and assume that neither $U \mid A$ nor $U \mid A^{\prime}$ is isomorphic to $U$. Then there exist disjoint, finite subsets $F_{1}, F_{2}$ of $A$ and $F_{1}^{\prime}, F_{2}^{\prime}$ of $A^{\prime}$ which satisfy: (i) if $v$ is connected in $U$ to every member of $F_{1}$ and to no member of $F_{2}$, then $v \in A$, and (ii) if $v$ is connected in $U$ to every member of $F_{1}^{\prime}$ and to no member of $F_{2}^{\prime}$, then $v \in A^{\prime}$. But $F_{1} \cup F_{1}^{\prime}$ and $F_{2} \cup F_{2}^{\prime}$ are disjoint, so there is a vertex $v$ which is connected in $U$ to every member of $F_{1} \cup F_{1}^{\prime}$ and to no member of $F_{2} \cup F_{2}^{\prime}$. This implies that $v \notin A \cup A^{\prime}$, which is a contradiction.

It follows immediately from Theorem 1.5 that if $A \subset|U|$ and 
$|U|-A$ is finite, then $U \mid A \cong U$. Also, using 1.5.a and the vertex symmetry of $U$ we note that $U^{v} \cong(\bar{U})^{v}$, for any $v \in|U|$. Then since $\left|U^{v}\right|$ and $\left|(\bar{U})^{v}\right|$ form a partition of $|U-v|$ it follows that $U^{v} \cong U$ for every $v \in|U|$.

Recall that two graphs $H_{1}, H_{2}$ with the same vertex set are called edge disjoint if $R\left(H_{1}\right) \cap R\left(H_{2}\right)=\varnothing$. If $\mathscr{F}$ is a family of graphs with a common vertex set $A$, then the union of $\mathscr{F}$ is the graph whose vertex set is $A$ and whose edge relation is $\bigcup\{R(H) \mid H \in \mathscr{F}\}$. $A$ spanning subgraph of $G$ is a graph $H$ which satisfies $|H|=|G|$ and $R(H) \subset R(G)$.

THEOREM 1.6. There is a family $\left\{H_{i} \mid i \in N\right\}$ of pairwise edge disjoint graphs (all with vertex set $N$ ) such that if $|G|=N, R\left(H_{i}\right) \subset R(G)$ and $R\left(H_{j}\right) \cap R(G)=\varnothing$ (for some $i, j \in N$ ) then $G \cong U$.

Proof. Let $\left\{\left(P_{n}, Q_{n}, f(n), g(n)\right) \mid n \in N\right\}$ be an enumeration of all quadruples $(A, B, i, j)$ in which $A, B$ are disjoint, finite subsets of $N$ and $i, j \in N$. Let $v_{0}<v_{1}<\cdots$ be a sequence in $N$ such that $v_{n}>\max \left(P_{n} \cup Q_{n}\right)$ for all $n \in N$. Define $H_{i}$, for each $i \in N$, by letting $\left|H_{i}\right|=N$ and letting $R\left(H_{i}\right)$ consist of all pairs of vertices $\left(w, v_{n}\right)$ and $\left(v_{n}, w\right)$ such that $f(n)=i$ and $w \in P_{n}$ or $g(n)=i$ and $w \in Q_{n}$.

Suppose $|G|=N$ and, for some $i, j \in N, G$ satisfies $R\left(H_{i}\right) \subset R(G)$ and $R\left(H_{j}\right) \cap R(G)=\varnothing$. Let $F_{1}, F_{2}$ be disjoint, finite subsets of $|G|$. Choose $n$ so that $P_{n}=F_{1}, Q_{n}=F_{2}, f(n)=i$ and $g(n)=j$. Then $v_{n}$ is connected in $H_{i}$ (and thus in $G$ ) to every member of $F_{1}$. Also $v_{n}$ is connected in $H_{j}$ (and thus not in $G$ ) to every member of $F_{2}$. This shows that $G$ satisfies condition (A) and therefore $G$ is isomorphic to $U$.

In particular, Theorem 1.6 asserts that the union of the family $\left\{H_{i} \mid i>0\right\}$ is isomorphic to $U$. Thus there exists a family $\left\{G_{i} \mid i \in N\right\}$ of pairwise edge disjoint spanning subgraphs of $U$ which satisfies (i) the union of the family is $U$, and (ii) if $G$ is any spanning subgraph of $U$ such that $R\left(G_{i}\right) \subset R(G)$, for some $i \in N$, then $G \cong U$.

Recall that a (one-way) Hamiltonian path for a graph $G$ (with $\left.c(G)=\boldsymbol{\aleph}_{0}\right)$ is a bijection $\tau$ from $N$ onto $|G|$ such that for each $n$, $\tau(n)$ and $\tau(n+1)$ are connected in $G$. The path $\tau$ will be called totally symmetric if the function sending $\tau(n)$ to $\tau(n+1)($ each $n \in N)$ is an embedding of $G$ into itself.

THEOREM 1.7. There exists a totally symmetric, one-way Hamiltonian path for $U$.

Proof. Let $\left\{P_{n} \mid n \in N\right\}$ be an enumeration of all finite subsets of $N$, with the properties: 
(i) $P_{n} \subset\{0, \cdots, n\}$ for each $n \in N$, and (ii) each finite subset of $N$ occurs in the list $\left\{P_{n} \mid n \in N\right\}$ infinitely often. For $n \in N$ define

$$
a_{n}=2+\frac{n(n+1)}{2}
$$

so that $a_{0}=2$ and $a_{n+1}=a_{n}+n+1$. Construct a chain $Q_{0} \subset Q_{1} \subset \cdots$ of finite subsets of $N-\{0\}$ by letting $Q_{0}=\{1\}$ and (for $n \geqq 0$ )

$$
Q_{n+1}=Q_{n} \bigcup\left\{a_{n+1}-k \mid k \in P_{n}\right\} \text {. }
$$

If $k \in P_{n}$ then $0 \leqq k \leqq n$ so that

$$
a_{n}+1=a_{n+1}-n \leqq a_{n+1}-k \leqq a_{n+1} .
$$

It follows, by induction on $n$, that $Q_{n} \subset\left\{0, \cdots, a_{n}\right\}$ and

$$
Q_{n+1}-Q_{n} \subset\left\{a_{n}+1, \cdots, a_{n+1}\right\} \text {. }
$$

Now let $A=\bigcup\left\{Q_{n} \mid n \in N\right\}$ and construct a graph $G$ with $|G|=$ $N$ and $R(G)=\{(m, n)|| m-n \mid \in A\}$. Since $1 \in A$, it is obvious that $G$ has a totally symmetric (one-way) Hamiltonian path. Thus it sufficies to prove that $G$ satisfies condition $(A)$, so that $U \cong G$.

If $F_{1}, F_{2}$ are disjoint, finite subsets of $N$, we may choose $n$ large enough so that $P_{n}=F_{1}$ and $F_{1} \cup F_{2} \subset\{0, \cdots, n\}$. For each $0 \leqq k \leqq n$ the construction of $Q_{n+1}$ insures that

$$
a_{n+1}-k \in Q_{n+1} \longleftrightarrow k \in F_{1} \text {. }
$$

But since $A \cap\left\{0, \cdots, a_{n+1}\right\}=Q_{n+1}$, it follows that

$$
a_{n+1}-k \in A \longleftrightarrow k \in F_{1} \text {. }
$$

Thus $a_{n+1}$ is connected in $G$ to every member of $F_{1}$ and to no member of $F_{2}$. That is, $G$ satisfies condition (A) and the proof is complete.

REMARK. Let $Z$ be the set of all the integers and $A$ the set constructed in the proof of Theorem 1.7. Define a graph $H$ with $|H|=Z$ by letting

$$
R(H)=\{(a, b) \mid a, b \in Z \text { and }|a-b| \in A\} .
$$

Evidently the functions $f$, sending $a$ to $a+1$, and $g$, sending $a$ to $-a$, are automorphisms of $H$. Moreover, since $1 \in A$, the identity function from $Z$ to $|H|$ defines a two-way Hamiltonian path for $H$. Finally, if $F_{1}, F_{2}$ are disjoint, finite subsets of $|H|$, choose $k$ large enough so that $f^{k}\left(F_{1} \cup F_{2}\right) \subset N$, and let $b \in N$ be connected in $H$ to every member of $f^{k}\left(F_{1}\right)$ and to no member of $f^{k}\left(F_{2}\right)$. (Choose $b$ using the fact that $H \mid N \cong U$, as proved above.) Then $f^{-k}(b)$ is connected in 
$H$ to every vertex in $F_{1}$ and to no vertex in $F_{2}$. That is, $H$ satisfies condition $(A)$ and is thus isomorphic to $U$.

This may be summarized by stating that $U$ has a totally symmetric, two-way Hamiltonian path. In particular, note that $U$ has an automorphism with a single orbit.

2. This section is devoted to a family $\left\{G_{p} \mid p \geqq 3\right\}$ of induced subgraphs of $U$, defined by letting

$$
\begin{aligned}
\left|G_{p}\right|= & \{m \mid m \in N \text { and there is no finite set } A \subset N \\
& \text { with } \left.m=\max A \text { and } U \mid A \cong K_{p}\right\},
\end{aligned}
$$

for each integer $p \geqq 3$. It follows that $G_{p} \subset G_{p+1} \subset U(p \geqq 3)$. and that $U$ is the union of the chain of graphs $\left\{G_{p} \mid p \geqq 3\right\}$. In addition, $G_{p}$ satisfies the following condition, analogous to (A).

$\left(A_{p}\right) \quad$ ( i ) $G$ does not admit $K_{p}$,

(ii) if $F_{1}, F_{2}$ are disjoint, finite sets of vertices of $G$ and $G \mid F_{1}$ does not admit $K_{p-1}$, then there is another vertex which is connected in $G$ to every member of $F_{1}$ and to no member of $F_{2}$.

Lemma 2.1. For each $p \geqq 3, G_{p}$ satisfies condition $\left(A_{p}\right)$.

Proof. It is obvious that $G_{p}$ satisfies (i). Suppose $F_{1}, F_{2}$ are disjoint, finite subsets of $\left|G_{p}\right|$ and that $G_{p} \mid F_{1}$ does not admit $K_{p-}$. Since $U$ satisfies $\left(A^{\prime}\right)$ we may choose $v \in|U|$ which satisfies $v>\max$ $\left(F_{1} \cup F_{2}\right)$ and

$$
F_{1}=\{w \mid w<v \quad \text { and } \quad(w, v) \in R(U)\} .
$$

It suffices to observe that $U\left|F_{1}=G_{p}\right| F_{1}$ dose not admit $K_{p-1}$ and therefore $v \in\left|G_{p}\right|$.

Lemma 2.2. Let $p \geqq 3$ and assume that $G$ satisfies condition $\left(A_{p}\right)$. Suppose also that $H$ is a finite graph which does not admit $K_{p}, v \in|H|$ and $f$ is an embedding of $H-v$ into $G$. Then $f$ can be extended to an embedding of $H$ into $G$.

THEOREM 2.3. For each $p \geqq 3, G_{p}$ is homogeneous, and admits exactly those finite graphs which do not admit $K_{p}$. Moreover, any graph $G$ (with $c(G)=\boldsymbol{\aleph}_{0}$ ) which satisfies condition $\left(A_{p}\right)$ is isomorphic to $G_{p}$.

Proof. Using Lemma 2.2, it can be shown by induction on $c(H)$ that if $G$ satisfies $\left(A_{p}\right)$ and $H$ is a finite graph which does not admit $K_{p}$, then $G$ admits $H$. That is, any graph which satisfies $\left(A_{p}\right)$ admits 
exactly those finite graphs which do not admit $K_{p}$.

It follows by Theorem 1.2 that if $c(G)=\boldsymbol{\aleph}_{0}$ and $G$ satisfies $\left(A_{p}\right)$ then $G$ is homogeneous. (In particular, by Lemma 2.1, $G_{p}$ is homogeneous.) Finally, by Theorem 1.3.b, any such $G$ is isomorphic to $G_{p}$.

The following result is an immediate consequence of Theorem 1.3.a and Theorem 2.3, and answers a question raised (for $p=3$ ) by Erdös and Hajnal [3, p. 121].

Corollary 2.4. For each $p \geqq 3, G_{p}$ is a universal graph in the class of countable graphs which do not admit $K_{p}$.

COROLlary 2.5. Let $p \geqq 3$.

(a) If $A \subset\left|G_{p}\right|$ and $\left|G_{n}\right|-A$ is finite, then $G_{p} \mid A \cong G_{p}$

(b) If $v \in\left|G_{p+1}\right|$ then $\left(G_{p+1}\right)^{v} \cong G_{p}$.

Proof. (a) If $F_{1}, F_{2}$ are disjoint, finite subsets of $A$ and $G_{p} \mid F_{1}$ does not admit $K_{p-1}$, then there are, in fact, infinitely many vertices in $\left|G_{p}\right|$ which are connected to every member of $F_{1}$ and to no member of $F_{2}$. Since $\left|G_{p}\right|-A$ is finite, this shows that $G_{p} \mid A$ satisfies $\left(A_{p}\right)$.

(b) Suppose $H$ is a finite graph satisfying $H \subset\left(G_{p++1}\right)^{v}$ and suppose that $f$ is an embedding of $H$ into $\left(G_{p+1}\right)^{v}$. Since $\mathrm{G}_{p+1}$ is homogeneous, there is an automorphism $g$ of $G_{p+1}$ such that $g$ extends $f$ and $g(v)=v$. Thus $g$ determines an automorphism of $\left(G_{p+1}\right)^{v}$ which extends $f$. This shows that $\left(G_{p+1}\right)^{v}$ is homogeneous. The fact that $\left(G_{p+1}\right)^{\prime \prime}$ and $G_{p}$ are isomorphic follows from Theorems 1.3.b and 2.3 and the observation that $\left(G_{p+1}\right)^{v}$ admits a finite graph $H$ if and only if $G_{p \rightarrow 1}$ admits the graph obtained from $H$ by adding a new vertex connected to every member of $|H|$.

Note that for each $v \in\left|G_{3}\right|$ the graph $\left(G_{3}\right)^{v}$ is infinite, with no two vertices connected.

The analogue of Corollary 1.5.b for $G_{p}$ is false, as can be seen by considering the partition of $\left|G_{p}\right|$ determined by $\left|\left(G_{p}\right)^{v}\right|$ and its complement. (Also see $\S 4$.)

If $H$ is a spanning subgraph of $G_{p}(p \geqq 3)$ and $H \neq G_{p}$, then $H$ cannot be isomorphic to $G_{p}$. For there must be vertices $a, b$ in $\left|G_{p}\right|$ which are connected in $G_{p}$ but not in $H$. If $H \cong G_{p}$ then there exists $A \subset\left|G_{p}\right|$ so that $H \mid A \cup\{a\}$ and $H \mid A \cup\{b\}$ are isomorphic to $K_{p-1}$. But this would imply that $G_{p} \mid A \cup\{a, b\} \cong K_{p}$, which is impossible. 
In particular, the analogue for $G_{p}$ of Theorem 1.6 is false. Corresponding to Theorem 1.7 are the following two results.

THEOREM 2.6 There exists a totally symmetric (one-way) Hamiltonian path for $G_{3}$.

Proof. Let the sequence $\left\{P_{n} \mid n \in N\right\}$ be as in the proof of Theorem 1.7, and construct a chain $Q_{0} \subset Q_{1} \subset \cdots$ of finite subsets of $N-\{0\}$ as follows. Let $Q_{0}=\{1\}$; for $n \geqq 0$, if there exist $a, b \in P_{n}$ so that $0<|a-b| \in Q_{n}$, then let $Q_{n+1}=Q_{n}$. Otherwise let

$$
Q_{n+1}=Q_{n} \bigcup\left\{3^{n+1}-k \mid k \in P_{n}\right\} .
$$

Recalling that $P_{n} \subset\{0, \cdots, n\}$, it follows that $Q_{n} \subset\left\{0, \cdots, 3^{n}\right\}$ and $Q_{n+1}-Q_{n} \subset\left\{3^{n}+1, \cdots, 3^{n+1}\right\}$. Let $A=\bigcup\left\{Q_{n} \mid n \in N\right\}$ and construct a graph $G$, as in the proof of Theorem 1.7, by letting $|G|=N$ and

$$
R(G)=\{(m, n)|| m-n \mid \in A\} \text {. }
$$

As before, it suffices to prove that this graph satisfies condition $\left(A_{3}\right)$.

Suppose that $F_{1}, F_{2}$ are disjoint, finite subsets of $|G|$ and that $G \mid F_{1}$ does not admit $K_{2}$. That is, if $a, b \in F_{1}$ and $a \neq b$ then $|a-b| \notin A$. Choose $n$ large enough so that $P_{n}=F_{1}$ and

$$
F_{1} \cup F_{2} \subset\{0, \cdots, n\} .
$$

Since $Q_{n} \subset A$ there do not exist $a, b \in P_{n}$ with $0<|a-b| \in Q_{n}$. Thus if $0 \leqq k \leqq n$ then $3^{n+1}-k \in Q_{n+1} \leftrightarrow k \in P_{n}$. It follows that $3^{n-1}$ is connected in $G$ to every member of $F_{1}$ and to no member of $F_{2}$.

Suppose next that $G$ admits $K_{3}$. It follows that there exist $0<a<b$ such that $G \mid\{0, a, b\} \cong K_{3}$. That is, $a, b$ and $b-a$ are in $A$. Let $n$ be the smallest integer for which $a \in Q_{n}$. If $b \in Q_{n}$ then $n \geqq 1$, and $a, b \in Q_{n}-Q_{n-1}$ (since $a<b$.) But then $a=3^{n}-c$ and $b=3^{n}-d$, for some $c, d \in P_{n-1}$. Moreover $c-d=b-a \in A$ and $0 \leqq d<c \leqq n-1$ so that $|c-d| \in Q_{n-1}$, contradicting the definition of $Q_{n}$. Therefore $b \notin Q_{n}$, and there exists $k \geqq n$ such that $b \in Q_{k+1}-Q_{k}$. If $b-a \in Q_{k+1}-Q_{\hat{k}}$ we obtain a contradiction as above, by considering $c, d \in P_{k}$ with $b=3^{k+1}-c$ and $b-a=3^{k+1}-d$.

Since $b-a<b \in Q_{k+1}$ and $b-a \in A$, it follows that $b-a$ must be in $Q_{k}$. Thus $a$ and $b-a$ are both $\leqq 3^{k}$ and therefore

$$
b \leqq 2 \cdot 3^{k}<3^{k+1}-k .
$$

But since $b \in Q_{k+1}-Q_{k}$, which implies that $3^{k+1}-k \leqq b \leqq 3^{k+1}$, this is a contradiction. That is, $G$ does not admit $K_{3}$.

This shows that $G$ satisfies the condition $\left(A_{3}\right)$ and therefore $G$ is isomorphic to $G_{3}$, completing the proof. 
As in the Remark following Theorem 1.7, it can be shown that $G_{3}$ has a totally symmetric, two-way Hamiltonian path. In particular, $G_{3}$ has an automorphism with a single orbit. In contrast, for the graphs $G_{p}$ with $p \geqq 4$ we have the following result.

Theorem 2.7. If $p \geqq 4$, then there is no automorphism of $G_{p}$ with a single orbit.

Proof. If otherwise, we can construct a graph $G$ with an automorphism $f$ such that $G \cong G_{p},|G|=Z$ and $f(\alpha)=a+1$ for all $a \in Z$. We let

$$
A=\{a \mid(a, 0) \in R(G)\}
$$

It then follows that

$$
R(G)=\{(a, b)|| a-b \mid \in A\} .
$$

Since $G_{p}$ admits $K_{p-2}$, there exist $a_{1}<\cdots<a_{p-2}$ in $|G|$ so that $G \mid\left\{a_{1}, \cdots, a_{p-2}\right\} \cong K_{p-2}$. That is, if $1 \leqq i<j \leqq p-2$ then $a_{j}-a_{i} \in A$. Since $G$ satisfies condition $\left(A_{p}\right)$ there exists $a \in|G|$ which is connected in $G$ to 0 but is not connected to any of the vertices $a_{i}-a_{j}$ (where $i \neq j$ ) and is distinct from them.

If $a_{i}$ is connected in $G$ to $a_{j}+a$, so that $\left|a_{j}+a-a_{i}\right|$ is in $A$, it follows that $a$ is connected to $a_{i}-a_{j}$. Thus $i=j$. (Conversely, $|a| \in A$, so that $a_{i}$ is connected to $a_{i}+a$.) If we let

$$
\mathrm{B}=\left\{a_{1}, \cdots, a_{p-2}, a_{1}+a, \cdots, a_{p-2}+a\right\},
$$

it follows that $G \mid B$ admits $K_{p-2}$ but not $K_{p-1}$ (recall that $p \geqq 4$ ). Thus there exists a vertex $k$ which is connected in $G$ to every member of $B$.

Consider $C=\left\{0, a, k-a_{1}, \cdots, k-a_{p-2}\right\}$. If $i \neq j$ then

$$
\left|\left(k-a_{i}\right)-\left(k-a_{j}\right)\right|=\left|a_{i}-a_{j}\right| \in A,
$$

so that

$$
G \mid\left\{k-a_{1}, \cdots, k-a_{p-2}\right\} \cong K_{p-2} .
$$

By the choice of $k,\left|k-a_{i}\right| \in A$ and $\left|k-a_{i}-a\right| \in A$. Thus each $k-a_{i}$ is connected in $G$ to 0 and to $a$. Since $a$ is connected to 0 in $G$ by choice, it follows that $G \mid C \cong K_{p}$. This contradicts the fact that $G \cong G_{p}$, and completes the proof.

REMARK. It is easy to show that if $G$ is a homogeneous graph, then so is $\bar{G}$. Thus the graphs $\bar{G}_{p}$ are all homogeneous, and evidently distinct from the graphs $U$ and $G_{p}(p \geqq 3$.) If $G$ is a homo- 
geneous graph, but not connected, the components of $G$ must be complete (consider the induced subgraphs with two vertices which are not connected) and pairwise isomorphic (since $G$ is vertex symmetric.) It is an interesting and apparently open question if there are any homogeneous graphs $G$ (with $c(G)=\boldsymbol{\aleph}_{0}$ ) which have $G$ and $\bar{G}$ connected, other than $U, G_{p}$ and $\bar{G}_{p}(p \geqq 3$.)

The existence of the graphs $G_{p}$ may be approached indirectly, by noting that the class $\mathscr{y}_{p}$ of all graphs which do not admit $K_{p}$ satisfies the amalgamation property of [7] (property D in [4].) Thus, in the language of [7], $G_{p}$ is the $\mathscr{L}_{p}$-homogeneous universal structure of cardinality $\boldsymbol{\aleph}_{0}$.

3. This section is concerned with the problem of embedding an infinite graph $H$ in $U$ (or in one of the graphs $G_{p}$ ) in such a way that automorphisms of $H$ extend to automorphisms of $U\left(G_{p}\right)$ In addition it is shown that each of these graphs has a maximal independent set $M$ whose permutations all extend uniquely to automorphisms.

THeorem 3.1. Let $H$ be a graph with $c(H)=\boldsymbol{\aleph}_{0}$. There exists an embedding of $H$ onto an induced subgraph $H^{\prime} \subset U$ such that each automorphim of $H^{\prime}$ extends uniquely to an automorphism of $U$.

Proof. Let $n_{1}<n_{2}<\cdots$ be an increasing sequence of positive integers. Construct a chain of graphs $H_{0} \subset H_{1} \subset H_{2} \subset \cdots$ by letting $H_{0}=H$ and continuing as follows. For $k \geqq 1$ obtain $\left|H_{k}\right|$ by adding to $\left|H_{k-1}\right|$ a new vertex $v(A, k)$ for each finite set $A \subset\left|H_{k-1}\right|$ such that $A \cap\left|H_{0}\right|$ has exactly $n_{k}$ elements. Each new vertex $v(A, k)$ is connected in $H_{k}$ to the vertices in $A$ and to no others. (Recall that $H_{k-1} \subset H_{k}$ is also required.) Define $K$ to be the union of the chain $\left\{H_{k} \mid k \geqq 0\right\}$ so that $H_{k} \subset K$ for each $k \geqq 0$ and, in particular, $H \subset K$.

If $F_{1}, F_{2}$ are disjoint, finite subsets of $|K|$, choose $k$ large enough so that $F_{1} \cup F_{2} \subset\left|H_{k-1}\right|$ and $F_{1} \cap\left|H_{0}\right|$ has at most $n_{k}$ elements. Since $\left|H_{0}\right|$ is infinite there is a set $B \subset\left|H_{0}\right|$ such that $B \cap F_{2}=\varnothing$, $F_{1} \cap\left|H_{0}\right| \subset B$ and $B$ has exactly $n_{k}$ elements. Letting $A=F_{1} \cup B$, it follows that $v(A, k)$ is a vertex in $H_{k}$ which is connected in $H_{k}$ (and thus in $K$ ) to every vertex in $F_{1}$ and to no vertex in $F_{2}$. This shows that $K$ satisfies condition (A). Since only countably many vertices are added at each stage of the construction of $K$, it follows that $K \cong U$.

Any automorphism $f$ of $H_{k-1}$ which satisfies $f\left(\left|H_{0}\right|\right)=\left|H_{0}\right|$ can be extended to an automorphism of $H_{k}$ by setting $f(v(A, k)=$ 
$v(f(A), k)$ (for each new vertex.) Moreover, since $f(v(A, k))$ must be connected in $H_{k}$ to the vertices in $f(A)$ and no others, this is the only possible way to extend such an $f$. Therefore, each automorphism of $H_{0}$ can be extended to an automorphism of $K$, and this extension is unique among automorphism of $K$ which leave each set $\left|H_{k}\right|$ invariant $(k>0$. $)$

But the members of $\left|H_{k}\right|$ are distinguished, among vertices of $K$, by virtue of being in $\left|H_{0}\right|$ or being connected in $K$ to at most $n_{k}$ elements of $\left|H_{0}\right|$. Thus any automorphism of $K$ which leaves $\left|H_{0}\right|$ invariant must also leave $\left|H_{k}\right|$ invariant, for each $k>0$. That is, each automorphism of $H\left(=H_{0}\right)$ has a unique extension to an automorphism of $K \cong U$, completing the proof.

COROLlary 3.2. There is a maximal independent set of vertices $M \subset|U|$ such that every permutation of $M$ extends uniquely to an automorphism of $U$.

Proof. Let $H$ be a graph with $\boldsymbol{\aleph}_{0}$ vertices, no two connected, and carry out the construction in the proof of Theorem 3.1. Set $M=\left|H^{\prime}\right| \subset|U|$ and note that every permutation of the set $M$ is an automorphism of $H^{\prime}$, and thus extends uniquely to an automorphism of $U$. Since $n_{k}>0$ (for $k \geqq 1$ ) each vertex in $|K|-|H|$ is connected to at least one member of $|H|$ in $K$. It follows that $M$ is a maximal independent set of vertices in $|U|$ as desired.

To extend Theorem 3.1 to the homogeneous graphs $G_{p}$ requires a modification of the construction given above. Fix $p \geqq 3$ and let $H$ be any graph, with $c(H)=\boldsymbol{\aleph}_{0}$, which does not admit $K_{p}$. Construct a chain $\left\{H_{k} \mid k \geqq 0\right\}$ by letting $H_{0}=H$ and proceeding as above, except that $v(A, k)$ is a vertex in $\left|H_{k}\right|-\left|H_{k-1}\right|$ only when $A \cap\left|H_{0}\right|$ has $n_{l}$ elements and $H_{k-1} \mid A$ does not admit $K_{p-1}$. ( $A$ any finite subset of $\left|H_{k-1}\right|, k \geqq 1$.) Letting $K$ be the union of the chain $\left\{H_{k}\right\}$, it is easy to see that the restriction on adding new vertices at each stage insures that $K$ does not admit $K_{p}$. Moreover, the same argument as above shows that each automorphism of $H\left(=H_{0}\right)$ extends uniquely to an automorphism of $K$.

It is not always true, however, that $K$ satisfies condition $\left(A_{p}\right)$. This difficulty can be overcome if we assume that $H$ satisfies

(B) if $F_{1} \subset|H|$ is finite, then there exists an infinite independent set $A \subset|H|-F_{1}$ such that no vertex in $F_{1}$ is connected in $H$ to any vertex in $A$.

Assume now that $H$ satisfies (B) and let $F_{1}, F_{2}$ be disjoint, finite subsets of $|K|$ such that $K \mid F_{1}$ does not admit $K_{p-1}$. Choose $k$ large enough so that $F_{1} \cup F_{2} \subset\left|H_{k-1}\right|$ and $F_{1} \cap\left|H_{0}\right|$ has at most $n_{k}$ elements. 
Let $F_{3} \subset\left|H_{0}\right|$ consist of $F_{1} \cap\left|H_{0}\right|$ together with every vertex in $\left|H_{0}\right|$ which is connected to some member of $F_{1}-\left|H_{0}\right|$. Since $F_{1}$ is finite and each vertex in $|K|-\left|H_{0}\right|$ is connected to only finitely many members of $\left|H_{0}\right|$, it follows that $F_{3}$ is a finite set. Applying condition (B), there exists an infinite independent set $A^{\prime}$ in $H_{0}$ such that $A^{\prime} \cap F_{3}=\varnothing$ and no vertex in $F_{3}$ is connected in $H_{0}$ to any vertex in $A^{\prime}$. In particular, $K \mid F_{1} \cup A^{\prime}$ does not admit $K_{p-1}$. Since $A^{\prime}$ is infinite, we may choose a set $B \subset\left(F_{1} \cup A^{\prime}\right) \cap\left|H_{0}\right|$ such that $B \cap F_{2}=\varnothing, F_{1} \cap\left|H_{0}\right| \subset B$ and $B$ has exactly $n_{k}$ elements. Letting $A=F_{1} \cup B$, it follows that $K \mid A$ does not admit $K_{p-1}$ and $A \cap\left|H_{0}\right|=B$ has $n_{k}$ elements. Thus $v(A, k)$ is a vertex in $K$ which is connected to every member of $F_{1}$ and to no member of $F_{2}$. That is, $K$ satisfies condition $\left(A_{p}\right)$ whenever $H$ satisfies condition (B).

THEOREM 3.3. Let $p \geqq 3$ and suppose $H$ is a graph with $c(H)=$ $\aleph_{0}$ which does not admit $K_{p}$. Then there is an embedding of $H$ onto an induced subgraph $H^{\prime} \subset G_{p}$ such that each automorphism of $H^{\prime}$ extends uniquely to an automorphism of $G_{p}$.

Proof. If $H$ satisfies (B) then the proof has been given above. Otherwise, extend $H$ to a graph $H^{\prime \prime}$ by adding a vertex $v^{\prime \prime}$ for each $v \in|H|$, connecting $v^{\prime \prime}$ only to $v$ in $H^{\prime \prime}$. Then $H \subset H^{\prime \prime}$ and $H^{\prime \prime}$ clearly does not admit $K_{p}$. If $F_{1}$ is a finite subset of $\left|H^{\prime \prime}\right|$ then letting $A=\left\{v^{\prime \prime}|v \in| H \mid-F_{1}\right\}-F_{1}$ shows that $H^{\prime \prime}$ satisfies condition (B). Finally, note that each automorphism $f$ of $H$ extends uniquely to an automorphism of $H^{\prime \prime}$ (by setting $f\left(v^{\prime \prime}\right)=\left(f(v)^{\prime \prime}\right.$.) The desired embedding of $H$ into $G_{p}$ is thus obtained by restricting to $H$ an appropriate embedding of $H^{\prime \prime}$ into $G_{p}$.

Corollary 3.4. For each $p \geqq 3$ there exists a maximal independent set of vertices $M \subset\left|G_{p}\right|$ such that every permutation of $M$ extends uniquely to an automorphism of $G_{p}$.

Proof. Prooceed as in the proof of Corollary 3.2, noting that the graph $H$ with $\boldsymbol{\aleph}_{0}$ vertices, no two connected, satisfies condition (B).

Theorem 3.5. Let $G$ be $U$ or $G_{p}$ for some $p \geqq 3$ and let

$$
a_{1}, \cdots, a_{n} \in|G| \text {. }
$$

There is an automorphism $f$ of $G$ which has $a_{1}, \cdots, a_{n}$ as its only fixed points.

Proof. Let $H^{\prime}$ be $G \mid\left\{a_{1}, \cdots, a_{n}\right\}$. Obtain $H$ from $H^{\prime}$ by adding 
a set $C=\left\{v_{n} \mid n \in Z\right\}$ of new vertices, but without adding any new edges. Obviously $H$ can be embedded in $G$ and $H$ satisfies (B). Let $c\left(H^{\prime}\right)<n_{1}<n_{2}<\cdots$ and using the sequence $\left\{n_{k}\right\}$ carry out the appropriate construction (as in the proof of Theorem 3.1 or Theorem 3.3.) We obtain a graph $K$ which is isomorphic to $G$ and satisfies $H \subset K$. Moreover, $K$ has an automorphism $f$ which satisfies $f(v)=v$ (if $v$ is one of $a_{1}, \cdots, a_{n}$ ) and $f\left(v_{n}\right)=v_{n+1}$ (if $n \in Z$ ). If $v=v(A, k)$ is any member of $|K|-|H|$, suppose $f(v)=v$. It follows that $f(A)=A$, and hence that $f(A \cap|H|)=A \bigcap|H|$. Now $A \cap|H|$ has $n_{k}>c\left(H^{\prime}\right)$ elements, so that $A \cap C \neq \varnothing$. Moreover, $f(A \cap C)=A \cap C$, which implies that $A \supset C$, contradicting the fact that $A$ is a finite set. Thus $f$ has no fixed points in $|K|-|H|$ and therefore has only $a_{1}, \cdots, a_{n}$ as fixed points. Finally note that there is an isomorphism $g$ of $K$ onto $G$ so that $g(v)=v$ if $v \in\left\{a_{1}, \cdots, a_{n}\right\}$. The automorphism $g \circ f \circ g^{-1}$ of $G$ has as its fixed points only $a_{1}, \cdots, a_{n}$, and is therefore the desired function.

4. It is well known that there are finite graphs of arbitrarily large chromatic number which do not admit $K_{3}$ (eg. [1].) Thus for each $p \geqq 3$ the graph $G_{p}$ has chromatic number $\aleph_{0}$. This may be expressed by saying that if $\left|G_{p}\right|=A_{1} \cup \cdots \cup A_{n}$ then for some $j=1, \cdots, n$ $G_{p} \mid A_{j}$ admits $K_{2}$. The results of this section amount to a strengthering of this fact.

THEOREM 4.1. Let $p \geqq 3$ and suppose $\left|G_{p}\right|=A_{1} \bigcup A_{2}$. Then either there exists $B \subset A_{1}$ such that $A_{1}-B$ is finite and $G_{p} \mid B \cong G_{p}$ or $G_{p} \mid A_{2}$ admits every finite graph which does not admit $K_{p}$.

Proof. Let $A_{1}, A_{2}$ be as above for $G_{p}$ and suppose that the desired set $B$ does not exist. Construct a sequence $\left\{\left(C_{n}, D_{n}\right) \mid n \geqq 1\right\}$, where $C_{n}, D_{n}$ are disjoint, finite subsets of $A_{1}$ (for each $n \geqq 1$ ) as follows. Since $G_{p} \mid A_{2}$ is not isomorphic to $G_{p}$, it fails to satisfy condition $\left(A_{p}\right)$. Thus there exist disjoint, finite subsets $\left(C_{1}, D_{1}\right)$ of $A_{1}$ such that $G_{p} \mid C_{1}$ does not admit $K_{p-1}$ and every vertex in $\left|G_{p}\right|$ which is connected to every member of $C_{1}$ and to no member of $D_{1}$ lies in $A_{2}$.

Assuming that $\left(C_{1}, D_{1}\right), \cdots,\left(C_{n}, D_{n}\right)$ have been constructed, let $E_{n}=\bigcup\left\{C_{j} \bigcup D_{j} \mid j=1, \cdots, n\right\}$ so that $E_{n}$ is a finite subset of $A_{1}$. Since $G_{p} \mid A_{1}-E_{n}$ is not isomorphic to $G_{p}$ there exist disjoint, finite subsets $\left(C_{n+1}, D_{n+1}\right)$ of $A_{1}-E_{n}$ such that $G_{p} \mid C_{n+1}$ does not admit $K_{p-1}$ and every vertex in $\left|G_{p}\right|$ which is connected to every member of $C_{n+1}$ and to no member of $D_{n+1}$ lies in $A_{2} \cup E_{n}$.

Now let $H$ be any finite graph which does not admit $K_{p}$ and 
suppose $|H|=\left\{a_{1}, \cdots, a_{n}\right\}$. For convenience assume that $|H| \cap\left|G_{p}\right|=$ $\varnothing$. Construct a graph $G$ with vertex set $|G|=|H| \cup E_{n}$ so that $G|(|H|)=H, G| E_{n}=G_{p} \mid E_{n}$ and each $a_{j}$ in $|H|$ is connected in $G$ to every element of $C_{j}$ and to no element of $E_{n}-C_{j}$. If $G \mid F \cong K_{p}$, then $F \cap|H| \neq \varnothing$ and $F \cap E_{n} \neq \varnothing$. Since each vertex in $E_{n}$ is connected in $G$ to at most one member of $|H|$ it follows that

$$
F \cap|H|=\left\{a_{j}\right\} \text { (for some } j=1, \cdots, n \text { ) and } F \cap E_{n} \subset C_{j} \text {. }
$$

That is, $G \mid C_{j}\left(=G_{p} \mid C_{j}\right)$ admits $K_{p-1}$, which is a contradiction. Therefore $G$ does not admit $K_{p}$.

Since $G_{p}$ is homogeneous, there is an embedding $f$ of $G$ into $G_{p}$ such that $f(v)=v$ for each $v \in E_{n}$. Therefore $f\left(a_{j}\right) \notin E_{n}$ (for each $j=$ $1, \cdots, n)$ and $f\left(a_{j}\right)$ is connected in $G_{p}$ to every vertex in $C_{j}$ and to no vertex in $D_{j}$. By the construction of $\left(C_{j}, D_{j}\right)$ it follows that $f\left(a_{j}\right) \in A_{2}$. That is, $f$ maps $H$ into $G_{p} \mid A_{2}$, showing that $G_{p} \mid A_{2}$ admits every finite graph which does not admit $K_{p}$.

COROLlary 4.2. Let $p \geqq 3$ and suppose that $\left|G_{p}\right|=A_{1} \cup \cdots \cup A_{n}$. Then for some $j=1, \cdots, n$ the graph $G_{p} \mid A_{j}$ admits every finite graph which does not admit $K_{p}$.

Proof. By induction on $n$, using Theorem 4.1.

We raise the question of whether or not the conclusion of Corollary 4.2 can be strengthened to read: " $G_{p} \mid A_{j}$ admits $G_{p}$, for some $j=1, \cdots, n . "$ ?

Corollary 4.2 is equivalent to the following result of Folkman [5] concerning finite graphs, which he proved by entirely different methods.

CoRollary 4.3. (Folkman) Let $p \geqq 3, n \geqq 2$ and suppose $G$ is any finite graph which does not admit $K_{p}$. There exists a finite graph $H$, which also does not admit $K_{p}$, such that if $|H|=A_{1} \cup \cdots \cup A_{n}$, then for some $j=1, \cdots, n, H \mid A_{j}$ admits $G$.

The proof of this equivalence is a standard application of (for example) König's Infinity Lemma, as in the proof of the Erdös-de Bruijn Theorem which states that an infinite graph $G$ has chromatic number $\geqq k$ if and only if it has a finite induced subgraph with chromatic number $\geqq k(k \in N)$. Thus the details will be omitted.

F. Galvin has raised the question of whether or not an "edge coloring" version of Corollary 4.3 holds when $p=3$. (See [3] for a 
discussion of this and related problems.) It seems possible that further investigation of $G_{3}$ might shed some light on this problem. The author is indebted to Fred Galvin for his useful comments on an earlier version of this paper.

\section{REFERENCES}

1. Blanche Descartes, Solution to advanced problem 4526, Amer. Math. Monthly, 61 (1954), 352.

2. P. Erdös, and A. Renyi, Asymmetric graphs, Acta Math. Acad. Sci. Hungar. 14 (1963), 295-315.

3. P. Erdös and A. Hajnal, Problems and results in finite and infinite combinatorial analysis, Annals of the New York Academy of Sciences (International Conference on Combinatorial Mathematics) 175, article 1 (1970), 115-124.

4. Roland Fraiseé, Sur 1'extension aux relations de quelques proprietés des ordres, Ann. Sci. École Norm. Sup. (3) 71 (1954), 361-388.

5. J. Folkman, Graphs with monochromatic complete subgraphs in every edge coloring, SIAM J. Appl. Math., 18 (1970), 19-24.

6. B. Jónsson, Universal relational systems, Math. Scand. 4 (1956), 193-208.

7. _ـ_ Homogeneous universal relational systems, Math. Scand. 8 (1960), 137-142.

8. M. Morley, and R. Vaught, Homogeneous universal models, Math. Scand., 11 (1962), $37-57$.

9. R. Rado, Universal graphs and universal functions, Acta Arith., 9 (1964), 331-340. 10. - Universal graphs, in F. Harary, ed., A Seminar in Graph Theory, Holt, Rinehart and Winston (New York, 1967), 83-85.

Received February 17, 1971.

DUKE UNIVERSITY 



\section{PACIFIC JOURNAL OF MATHEMATICS}

\section{EDITORS}

H. SAMELSON

Stanford University

Stanford, California 94305

C. R. Hовву

University of Washington

Seattle, Washington 98105
J. DUGUNDJI

Department of Mathematics

University of Southern California

Los Angeles, California 90007

RICHARD ARENS

University of California

Los Angeles, California 90024

\section{ASSOCIATE EDITORS}
E. F. BECKENBACH
B. H. NeumanN
F. WOLF
K. YoSHIDA

\section{SUPPORTING INSTITUTIONS}

UNIVERSITY OF BRITISH COLUMBIA

CALIFORNIA INSTITUTE OF TECHNOLOGY

UNIVERSITY OF CALIFORNIA

MONTANA STATE UNIVERSITY

UNIVERSITY OF NEVADA

NEW MEXICO STATE UNIVERSITY

OREGON STATE UNIVERSITY

UNIVERSITY OF OREGON

OSAKA UNIVERSITY

UNIVERSITY OF SOUTHERN CALIFORNIA
STANFORD UNIVERSITY

UNIVERSITY OF TOKYO

UNIVERSITY OF UTAH

WASHINGTON STATE UNIVERSITY

UNIVERSITY OF WASHINGTON

AMERICAN MATHEMATICAL SOCIETY CHEVRON RESEARCH CORPORATION NAVAL WEAPONS CENTER 


\section{Pacific Journal of Mathematics}

\section{Vol. 38, No. $1 \quad$ March, 1971}

Bruce Alan Barnes, Banach algebras which are ideals in a Banach algebra ..... 1

David W. Boyd, Inequalities for positive integral operators............... 9

Lawrence Gerald Brown, Note on the open mapping theorem .............. 25

Stephen Daniel Comer, Representations by algebras of sections over Boolean

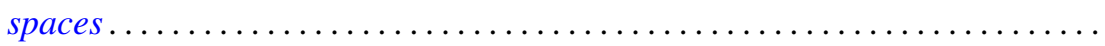

John R. Edwards and Stanley G. Wayment, On the nonequivalence of

conservative Hausdorff methods and Hausdorff moment sequences ........

P. D. T. A. Elliott, On the limiting distribution of additive functions $(\bmod 1) \ldots \ldots$

Mary Rodriguez Embry, Classifying special operators by means of subsets

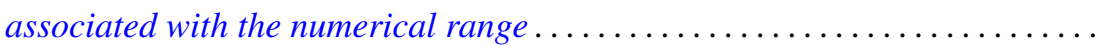

Darald Joe Hartfiel, Counterexamples to a conjecture of G. N. de Oliveira ......

C. Ward Henson, A family of countable homogeneous graphs...............

Satoru Igari and Shigehiko Kuratsubo, A sufficient condition for

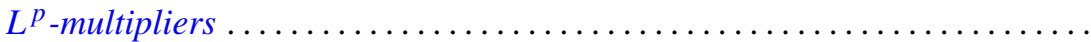

William A. Kirk, Fixed point theorems for nonlinear nonexpansive and

generalized contraction mappings............................

Erwin Kleinfeld, A generalization of commutative and associative rings ...... 95

D. B. Lahiri, Some restricted partition functions. Congruences modulo $11 \ldots \ldots 103$

T. Y. Lin, Homological algebra of stable homotopy ring $\pi *$ of spheres ....... 117

Morris Marden, A representation for the logarithmic derivative of a meromorphic function...........................

John Charles Nichols and James C. Smith, Examples concerning sum properties for metric-dependent dimension functions . .

Asit Baran Raha, On completely Hausdorff-completion of a completely

Hausdorff space.

M. Rajagopalan and Bertram Manuel Schreiber, Ergodic automorphisms and affine transformations of locally compact groups..........

N. V. Rao and Ashoke Kumar Roy, Linear isometries of some function

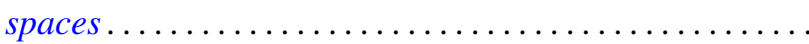

William Francis Reynolds, Blocks and F-class algebras of finite groups

Richard Rochberg, Which linear maps of the disk algebra are multiplicative ...

Gary Sampson, Sharp estimates of convolution transforms in terms of decreasing

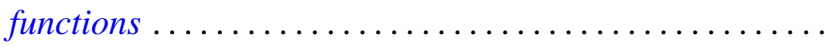

Stephen Scheinberg, Fatou's lemma in normed linear spaces

Ken Shaw, Whittaker constants for entire functions of several complex

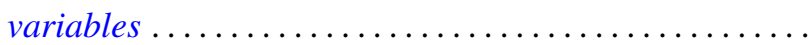

James DeWitt Stein, Two uniform boundedness theorems................ 251

$\mathrm{Li} \mathrm{Pi} \mathrm{Su,} \mathrm{Homomorphisms} \mathrm{of} \mathrm{near-rings} \mathrm{of} \mathrm{continuous} \mathrm{functions} \mathrm{.} \mathrm{.............} 261$

Stephen Willard, Functionally compact spaces, $C$-compact spaces and mappings of minimal Hausdorff spaces....................... 